\title{
Análisis de la maduración del juicio moral en el proceso de aprendizaje de la bioética
}

\author{
M. Teresa Delgado-Marroquín, Rogelio Altisent, Begoña Buil, Pablo Muñoz, M. Nieves Martín-Espíldora, \\ Pablo Rodríguez del Pozo
}

Objetivo. Medir el razonamiento moral de los estudiantes de medicina antes y después de recibir formación en bioética en dos ámbitos culturales diferentes (Zaragoza y Doha) y de los residentes al inicio de su residencia (MIR1), correlacionando el razonamiento moral con el ámbito cultural y el ambiente de aprendizaje.

Sujetos y métodos. Estudio observacional transversal del razonamiento moral con estudiantes de medicina y MIR1 y de intervención con seguimiento longitudinal del razonamiento moral antes y después de la formación en bioética, utilizando el test de razonamiento moral de Lind.

Resultados. Se obtuvieron 273 cuestionarios iniciales de estudiantes (200 de Zaragoza y 73 de Qatar) y 141 de MIR1. Se tiene información antes y después de 122 estudiantes ( $44,7 \%$ del total), una quinta parte de Qatar. Antes de bioética, la media del $C$-score era de 14,24 $\pm 8,698(n=273)$. Después, la media era de 14,30 $\pm 10,111(n=194)$. La diferencia media antes-después fue de $0,79 \pm 12,162(n=122)$. No hay diferencias del $C$-score por región, pero sí al dicotomizarlo en preconvencional $(<10)$ y convencional-posconvencional $(\geq 10): 49 \%<10$ en Qatar frente a 30\% en Zaragoza. También hay diferencias significativas en las respuestas dadas a los dos dilemas que conforman el test de razonamiento moral entre ambas regiones. Los MIR de Zaragoza muestran el mismo patrón de respuestas ante los dilemas que los estudiantes, pero su C-score es significativamente inferior (10,4 frente a 14,3).

Conclusiones. Se confirman las hipótesis iniciales, aunque conviene profundizar en esta línea de investigación durante más cursos académicos, o incluyendo otros campus universitarios de las mismas zonas culturales.

Palabras clave. Bioética. Educación médica. Razonamiento moral.

Division of Medical Ethics; Weill Cornell Medical College; Doha, Qatar (P. Rodríguez del Pozo). Grupo de Investigación en Bioética; Instituto de Investigación Sanitaria de Aragón; Cátedra de Profesionalismo y Ética Clínica; Universidad de Zaragoza (M.T. Delgado-Marroquín, R. Altisent, B. Buil, P. Muñoz, M.N. MartínEspíldora); Zaragoza, España.

Correspondencia: Prof. M. Teresa Delgado Marroquín. Centro de Salud Universitario Delicias Norte. Santa Orosia, 46. E-50010 Zaragoza.

E-mail: maitedelgadom@gmail.com

Conflicto de intereses: No declarado.

Competing interests: None declared.

(c) 2014 FEM

\section{Analysis of the maturation of moral judgment in the learning process of bioethics}

Aim. To measure the moral reasoning of medical students before and after training in bioethics in two different cultural settings (Zaragoza and Doha), as well as MIR1s in Zaragoza, correlating in both cases the possible changes in their moral reasoning with their cultural and learning environment.

Subjects and methods. Observational study of moral reasoning with medical students and MIR1 and intervention with longitudinal follow up moral reasoning before and after training in bioethics, using Georg Lind's Moral Judgement Test.

Results. There were 273 initial questionnaires of students (200 from Zaragoza, 73 from Qatar) and 141 MIR1. There were information before-after 122 students (44.7\%, of which $1 / 5$ from Qatar). Before bioethics training the average C-score was $14.24 \pm 8.698(n=273)$. After training, the average was $14.30 \pm 10.111(n=194)$. The mean difference before and after was $0.79 \pm 12.162(n=122)$. No C-score differences by region were found, but the dichotomization in preconventional $(<10)$ and conventional-postconventional showed differences between Qatar and Zaragoza ( $\geq 10): 49 \%<10$ versus 30\%, respectively). There were also significant regional differences in the responses to the two dilemmas that compose the Moral Judgement Test. The MIR of Zaragoza showed the same pattern of responses to the dilemmas than students but their C-score is significantly lower (10.4 versus 14.3).

Conclusions. We confirm the initial hypothesis, although this line of research should be furthered to more academic years and more university campuses.

Key words. Bioethics. Medical education. Moral judgement. 


\section{Introducción}

La práctica clínica necesita médicos capaces de resolver situaciones complejas en las que son imprescindibles tanto el juicio clínico como el juicio moral: proceso que permite reflexionar sobre los valores en conflicto y ordenarlos en una jerarquía justa. Desde la dimensión ética del compromiso profesional, la competencia moral consiste en la capacidad de movilizar recursos cognitivos y afectivos para solucionar conflictos morales [1] en el contexto clínico. Se necesita una práctica clínica reflexiva que incluya el compromiso personal y la adquisición de competencias para mejorar la calidad asistencial.

Una práctica clínica reflexiva estará relacionada con la incorporación de la bioética -entendida aquí como una forma de tomar decisiones y analizar situaciones mediante la deliberación [2]- a la práctica diaria. Ahora bien, ¿puede la bioética educar el juicio moral de los médicos? De ser así, ¿existe un método válido para medir el posible desarrollo del juicio moral?

Lawrence Kohlberg [3], discípulo de Jean Piaget, describió tres niveles de desarrollo de la capacidad de juicio moral [4], siguiendo una escala de maduración individual. El primer nivel de Kohlberg consiste en una moralidad preconvencional, en donde la percepción del bien y del mal está vinculada a las consecuencias físicas beneficiosas o dañinas de las acciones del sujeto. El segundo nivel lo conforma una moralidad convencional, en donde el sujeto vincula la bondad o maldad de sus actos a la adhesión a una regla o norma, aunque sin efectuar un juicio crítico sobre ella. El grado más alto de maduración está en el nivel posconvencional, en donde la libre convicción del sujeto lo lleva a ajustarse a una norma a la cual se adhiere por íntima convicción.

Si aplicamos esta doctrina a los estudiantes de medicina en sus prácticas clínicas o los residentes (MIR) en su propedéutica profesional:

- Para un estudiante o MIR que estuviera en un nivel preconvencional de desarrollo moral, actuar moralmente consistiría únicamente en evitar consecuencias negativas (daño al paciente, temor a una sanción).

- Un estudiante o MIR en el nivel convencional enfocaría la solución de los conflictos morales esforzándose no sólo en evitar el daño y la sanción, sino también en vivir de acuerdo con normas y definiciones socialmente aceptadas de lo que es ser un buen médico y un buen ciudadano.

- Por último, un estudiante o MIR en el nivel posconvencional enfocaría la solución de los conflictos morales de la práctica clínica desde la pers- pectiva de 'lo justo', esto es, podría ver que existen soluciones 'justas' según sus íntimas convicciones. En este nivel, el sujeto interpretará la norma para hacerla coincidir con su criterio.

Kohlberg postuló el uso de entrevistas estructuradas para la evaluación de la competencia para el juicio moral. Sobre aquellos primeros pasos, Georg Lind [5,6] logró elaborar un instrumento más práctico, el test del juicio moral -Moral Judgement Test (MJT)-, capaz de medir esta competencia. Lind propuso que en los procesos de decisión moral están presentes tanto aspectos cognitivos como aspectos afectivos que se involucran y forman parte de toda decisión. Del juego entre ambos aspectos surge la 'competencia para el juicio moral', un término que había empleado Kohlberg sin llegar a hacerlo operativo.

Los estudiantes de medicina y los MIR son personas que llevan 20 años en un sistema de formación continua y que han ido pasando por sucesivos procesos de selección, lo cual los hace poseedores de algunas características comunes: haber estado entre los mejores de su clase, haber logrado excelentes resultados académicos en el bachillerato, muy buenas notas en la selectividad o altas puntuaciones en el Medical College Admission Test (MCAT) -examen obligatorio para el ingreso a las facultades de medicina de las universidades estadounidenses$y$, en su caso, haber superado el examen MIR. Se trata, por tanto, de personas que pudiendo por méritos acceder casi a cualquier carrera, eligen medicina, una profesión de muy largo recorrido formativo (al menos 10 años) y con un alto componente altruista y de servicio social [7]. La revisión de la bibliografía confirma, fuera de nuestras fronteras, un elevado nivel de competencia para el razonamiento moral entre los estudiantes de medicina al inicio de su carrera, superior incluso al de otras disciplinas universitarias (media de C-score: 46 frente a 41 [6]). Lamentablemente, las investigaciones constatan también que la competencia para el juicio moral sufre entre los estudiantes de medicina una regresión estadísticamente significativa a medida que progresan en su carrera [8-11]. Esta regresión no se observa, sin embargo, entre los estudiantes de otras carreras $[7,10]$.

Las causas de esta regresión no han sido bien determinadas, aunque se ha señalado el impacto de los llamados currículos informal y oculto [10,12]. El currículo informal está dado por la manera no necesariamente pautada de relacionarse los profesores -individual y colectivamente- con los estudiantes; el currículo oculto consiste en los mensajes que implícitamente reciben los estudiantes sobre la es- 
tructura y la cultura organizativa de su facultad e institución sanitaria [13]. Estos elementos provocarían frustración entre los estudiantes, al no poder llevar a la práctica los valores que los condujeron a estudiar medicina; ello provocaría en definitiva la regresión en la capacidad para el juicio moral $[9,10$, $14,15]$. No hay información en nuestro medio sobre el punto de partida del juicio moral de los MIR y su evolución a lo largo de la residencia, ni sobre una posible propensión hacia el deterioro del juicio moral entre aquellas personas que se deciden a estudiar medicina.

Algunos estudios indican que los cursos de ética médica tienen un impacto positivo en el desarrollo de las competencias en el ámbito moral de los alumnos [15]. Sin embargo, estos estudios son pequeños y están limitados a los estudiantes de posgrado. Más recientemente, el propio Lind $[16,17]$ postula que no se trata de la formación en ética, sino de la participación activa en la toma de decisiones éticas lo que podría frenar la regresión de la capacidad de juicio moral de los estudiantes de medicina.

\section{Justificación}

La revisión de la bibliografía confirma diferencias en el valor de partida del juicio moral en función de distintos ámbitos culturales y sistemas de aprendizaje, así como una regresión significativa a medida que avanza el nivel de formación médica. Esta regresión puede ser menor entre los estudiantes y residentes que participan activamente en la formación en bioética. No hay estudios realizados en nuestro país al respecto, ni comparativos con otros ámbitos culturales que consideren una formación estructurada en bioética centrada en la participación activa y las necesidades concretas de los estudiantes y MIR.

Las hipótesis de partida son las siguientes:

- Es posible determinar de forma válida y fiable el nivel de maduración moral de los estudiantes y residentes de medicina y seguir longitudinalmente su evolución en el tiempo mediante pruebas test-retest con el MJT de Lind.

- La formación en bioética es determinante en la evolución del nivel de razonamiento moral de los médicos pre y posgraduados.

- El ámbito cultural influye en la evolución del nivel de razonamiento moral de los médicos pre y posgraduados.

Los objetivos de la investigación son:

- Medir el razonamiento moral de los estudiantes de medicina de primer ciclo, antes y después de recibir formación específica en bioética mediante el MJT de Lind, en dos ámbitos culturales diferentes (Zaragoza y Doha).

- Correlacionar el razonamiento moral con el ámbito cultural y el ambiente de aprendizaje en dos entornos diferentes: mediterráneo (España) e islámico (Qatar).

- Medir el razonamiento moral de los MIR al comienzo de su formación especializada en Zaragoza mediante el MJT de Lind.

- Estudiar si se confirma regresión en el razonamiento moral de los MIR respecto a los estudiantes y su relación con la formación en bioética.

- Objetivo secundario: valorar otras variables de influencia.

\section{Sujetos y métodos}

Se trata de un estudio de diseño cuantitativo en dos fases:

- Estudio observacional transversal del razonamiento moral con estudiantes de medicina y MIR al inicio de su formación.

- Estudio de intervención con seguimiento longitudinal del razonamiento moral de los estudiantes antes y después de su formación.

\section{Instrumento}

El MJT de Lind, en sus versiones validadas en castellano e inglés -con permiso del autor para fines docentes [17]-, consiste en la presentación de dos dilemas morales: el dilema del trabajador, en donde el sujeto debe juzgar el proceder de dos trabajadores que irrumpen de noche en su empresa para buscar evidencias favorables a compañeros injustamente despedidos, y el dilema del médico, en donde un galeno receta una alta dosis de morfina a petición de una paciente terminal que desea morir. El sujeto evalúa la solución general a estos dilemas en una escala que va de -3 a +3 (total desacuerdo-total acuerdo). A continuación, los sujetos deben juzgar si una serie de argumentos dados a favor y en contra de la solución general son aceptables. Estos argumentos representan diversos niveles de razonamiento moral.

La puntuación principal del MJT es el C-score, valor numérico continuo entre 0 y 100 . El C-score se obtiene tabulando mediante un algoritmo matemático las respuestas dadas al MJT. El algoritmo parte de la noción de que sólo se puede entender el significado de una respuesta del sujeto cuando se consideran otros juicios del mismo individuo. El C-score 
mide la capacidad de una persona para juzgar los argumentos de otros con relación a los estándares morales que ha aceptado como válidos para sí misma. Así, el sujeto obtendrá un alto C-score cuando su juicio sobre los argumentos a favor y en contra muestre consistencia. Se interpreta de la siguiente manera: valores de 0-9 corresponden a un C-score bajo, relacionados con un estadio de maduración moral preconvencional de Kohlberg; valores de 10-29, a un $C$-score medio, correspondiente a un estadio convencional, y valores $\geq 30$, un $C$-score alto o muy alto (estadio posconvencional).

\section{Población}

- Estudiantes: estudiantes de la Facultad de Medicina de la Universidad de Zaragoza (UNIZAR) y de la Facultad de Medicina de la Universidad de Cornell en su campus de Qatar (WCMC-Q), antes y después de la asignatura obligatoria de bioética en segundo curso (cursos académicos 2009 y 2010).

- MIR: residentes de primer año (MIR1) de todas las especialidades de las unidades docentes de Zaragoza (2010 y 2011).

\section{Muestra}

Alumnos que acudieron a clase los días de la administración de la prueba y respondieron voluntariamente el cuestionario MJT.

\section{Procedimiento}

Se solicitó consentimiento del profesor responsable y de los discentes para responder a la encuesta anónima (participación voluntaria) con el MJT y las variables demográficas de interés.

Se efectuaron sesiones de 15-20 minutos: 3-5 minutos para la lectura de la carta de presentación y explicaciones técnicas y 12-15 minutos para completar el cuestionario.

Para hacer posible el seguimiento longitudinal de las respuestas al test, se solicitó a cada participante que escribiera un código de identificación personal inequívoco y anónimo, fácil de recordar: UNIZAR y MIR1, la fecha de nacimiento (año, mes, día) y las dos primeras letras del segundo apellido del padre, o del apellido de soltera de la madre para las personas con un solo apellido. WCMC-Q: el número del móvil personal.

Se aseguró por escrito la confidencialidad de las respuestas obtenidas y la posibilidad de retirar el consentimiento a participar en el estudio.
El estudio recibió las necesarias autorizaciones para cumplir con la normativa sobre investigación con seres humanos.

\section{Procesamiento y análisis estadístico de los datos}

Se creó una base de datos en Excel con el algoritmo para el cálculo del C-score y el resto de las variables independientes. Los datos se introdujeron por un solo investigador que los revisó uno a uno para confirmar que la información almacenada no contenía errores. Las variables demográficas se codificaron de acuerdo con las tablas sugeridas por Lind en la bibliografía. Para el análisis estadístico se utilizó el programa SPSS v. 18.0 en castellano.

\section{Resultados}

\section{Resultados de los estudiantes de medicina}

Se obtuvieron 273 cuestionarios iniciales respondidos de forma voluntaria entre ambas regiones (200 de Zaragoza y 73 de Doha). Se perdió información por faltar la respuesta antes y después y, sobre todo, porque 151 participantes omitieron responder alguno de los 14 ítems del MJT. Es decir, se tiene información antes y después de 122 estudiantes $(44,7 \%$ del total, de los cuales una quinta parte eran de Qatar).

Antes del curso de bioética, la puntuación media del C-score era 14,24 $\pm 8,698(n=273)$. La distribución de la puntuación $C$-score no se ajustaba a la normalidad. Después de la formación, la puntuación media del $C$-score fue de 14,30 $\pm 10,111(n=194)$. La distribución tampoco se ajustaba a la normalidad.

La diferencia antes-después del C-score, en cambio, sí tiene una distribución normal: la media es de $0,79 \pm 12,162(n=122)$, con mayor desviación en las medidas de las mujeres, aunque las medianas son similares. No hay diferencias significativas y no hay correlación entre ambas distribuciones.

Para evaluar los factores que influyen en el cambio del C-score, se hizo un análisis de varianza con el cambio como variable dependiente y el sexo y la región como independientes. El resultado para ambas variables no fue significativo $(F=0,40, p=0,5291$ para el año, y $F=0,00, p=0,9683$ para el sexo).

Para conocer la influencia de la región (Doha, Zaragoza) se hizo un análisis no paramétrico (sobre las medianas, no sobre las medias, porque los datos no siguen una distribución normal). El test de Kruskal-Wallis no resultó significativo $\left(\chi^{2}=0,0449 ; p=\right.$ 0,8323). 
Respecto a las dos zonas geográficas, la muestra de Zaragoza presenta más mujeres (69\%), mientras que la de Qatar está más equilibrada y sólo el $54 \%$ son mujeres $\left(\chi^{2}=4,19 ; p=0,0406\right)$. La edad en Qatar es de 22 años (media y mediana), y en Zaragoza, de 19 años (test de Kruskal-Wallis significativo; $\chi^{2}=$ 96,46; $p=<0,0001)$. Al ser datos demográficos, se tomó toda la muestra, independientemente de la presencia o ausencia de medida de C-score antes y después. La mayor dispersión que se aprecia en Zaragoza puede deberse al mayor tamaño de la muestra. Las medianas son iguales en ambos grupos (Fig. 1).

Aunque la edad es significativamente más baja en Zaragoza, es difícil medir su influencia en el cambio en las puntuaciones del C-score porque la mayoría de sujetos tiene 19-20 años. Aunque haya diferencia de edad por región, no se aprecian diferencias del $C$-score por región, así que la influencia de la edad es nula o se ve anulada por otros factores (Fig. 2).

Dicotomizando los resultados del C-score en puntuaciones $<10$ (correspondientes a un estadio preconvencional de Kohlberg) y $\geq 10$ (estadio convencional o posconvencional), sí aparecen diferencias: hay más proporción de puntuaciones < 10 en Qatar que en Zaragoza (49\% frente a 30\%). Tras explorar un modelo con interacciones, se desechó, y en un modelo con factores principales, la única variable que explica la puntuación en el C-score es la región $\left(\chi^{2}=16,5 ;\right.$ g.l. $\left.=3 ; p=0,0009\right)$.

Además del indicador C-score, el MJT ofrece otros indicadores, el grado de acuerdo o de desacuerdo, medido a través de las puntuaciones a los dos dilemas sobre los que se sustenta el test: el dilema del trabajador y el dilema del médico.

En cuanto a la respuesta ante el dilema del trabajador, en global, los estudiantes de medicina antes de la actividad formativa muestran su acuerdo con lo hecho por los trabajadores (puntuaciones de 1 a 3 ) en el 70\% de los casos (el $57 \%$ eligen valores de 1 o 2 : poco de acuerdo o de acuerdo); por el desacuerdo o la neutralidad (puntuaciones de -3 a 0 ) se decanta el 30\%. La puntuación media es de 0,92 $\pm 1,573$, sin distribución normal. Después de la actividad formativa, los estudiantes se muestran de acuerdo (puntuaciones de 1 a 3) sólo en el 56\% de los casos (el $34,5 \%$ eligen un valor de 1: poco de acuerdo) y en desacuerdo o neutrales (puntuaciones de -3 a 0 ) en el $44 \%$ de los casos. La puntuación media es de $0,28 \pm 1,495$.

Con relación a la respuesta frente al dilema del médico, en global, los estudiantes de medicina antes de la actividad formativa se muestran de acuerdo o muy de acuerdo con el médico que prescribió
Figura 1. Cambio en la puntuación del C-score (después-antes) por región.

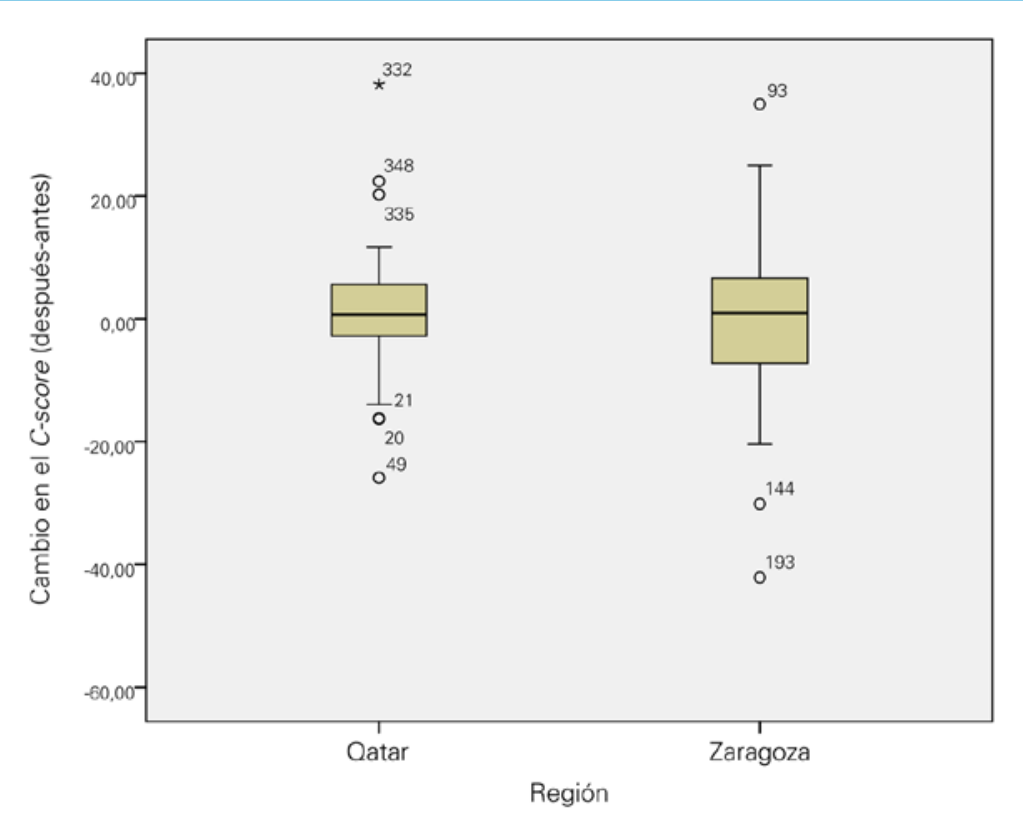

Figura 2. Cambio en la puntuación del C-score (después-antes) por edad, sexo y región.

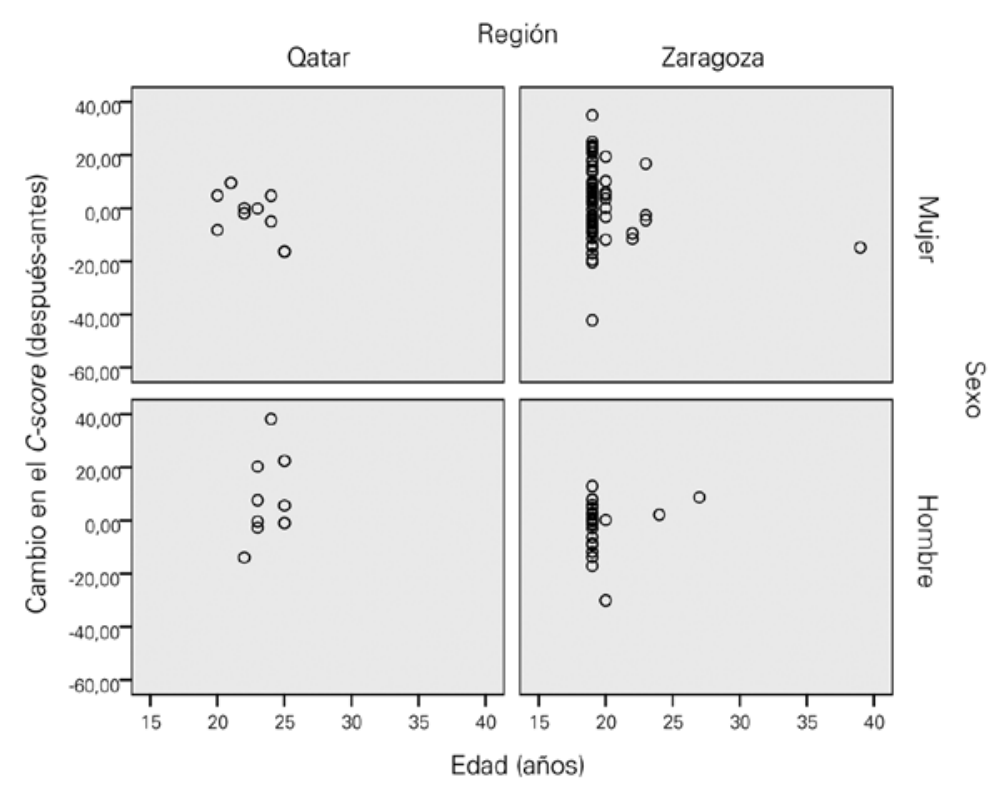

morfina (puntuaciones de 1 a 3 ) en el $71,5 \%$ de los casos (el $54,5 \%$ eligen valores de 2 o 3 ), y en desacuerdo o neutrales (puntuaciones de -3 a 0 ), en el 
Figura 3. Variación en la respuesta de los estudiantes de medicina ante los dilemas del médico y del trabajador antes y después de la formación en bioética.

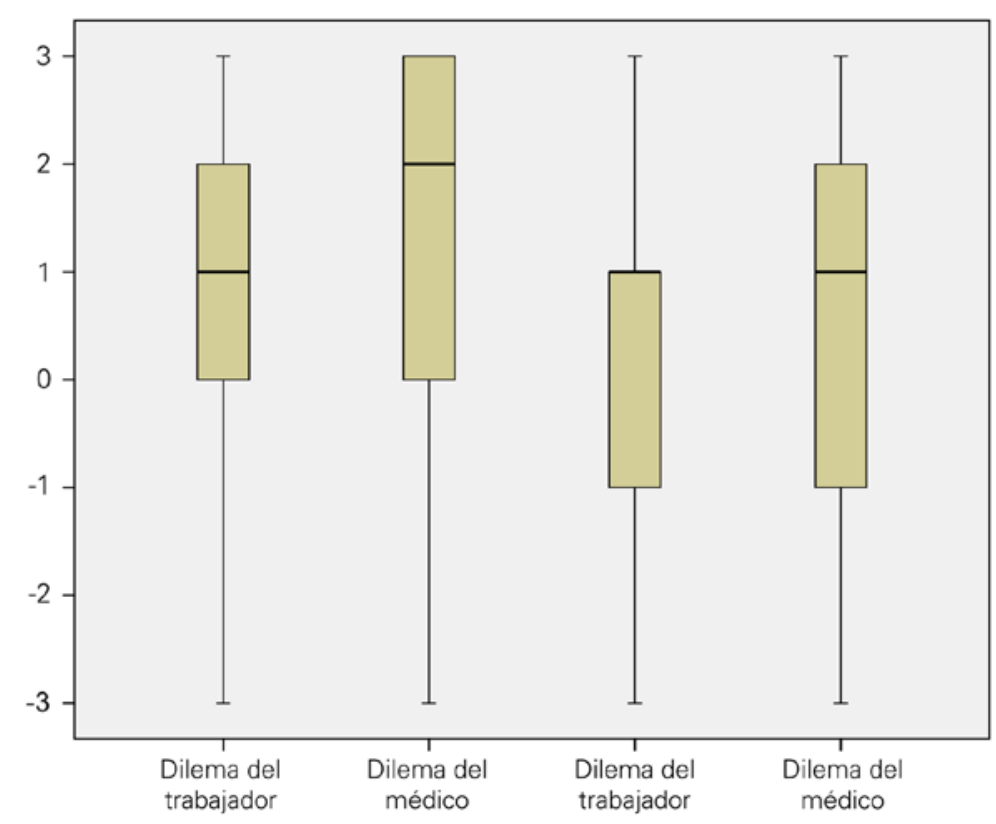

Figura 4. Opinión de los estudiantes de Qatar y Zaragoza ante los dilemas del trabajador y del médico del MJT antes y después de la formación en bioética.

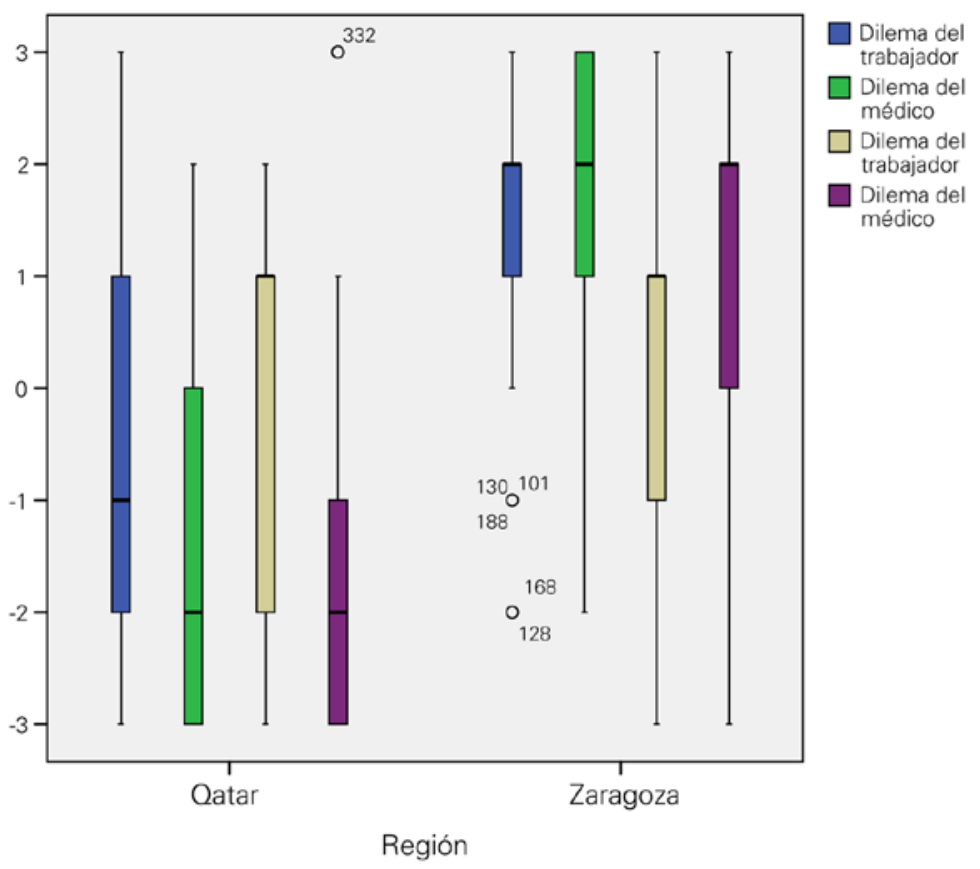

28,5\%. La puntuación media es de 1,08 $\pm 1,948$, sin distribución normal. Después de la actividad formativa, los estudiantes se muestran de acuerdo o muy de acuerdo (puntuaciones de 1 a 3 ) en el $56,5 \%$ de los casos (el $40 \%$ eligen valores de 2 o 3 ), y en desacuerdo o no se decantan (puntuaciones de -3 a 0), en el 43,5\%. La puntuación media es de 0,38 $\pm 2,019$.

El cambio mediano en el dilema del trabajador es de 1 punto y resulta significativo (test de signos; $M=24,5 ; p<0,0001)$. El cambio mediano en el dilema del doctor es de 0 puntos y resulta significativo (test de signos; $M=16,0 ; \mathrm{p}=0,0002$ ) (Fig. 3).

Al valorar los resultados en función de la región de procedencia, para el dilema del trabajador, se observa una diferencia no significativa aunque quizá se deba a la poca muestra en Qatar $(p=0,0590)$; sin embargo, sí se observa para el dilema del doctor (puntuaciones más altas en Zaragoza que en Qatar). En Qatar, las puntuaciones son en general más bajas (Fig. 4).

\section{Residentes de medicina}

Se obtuvo información válida de 141 (47\%) MIR1 de las unidades docentes 2 y 3 de Zaragoza (hospitales clínicos universitarios Lozano Blesa y Miguel Servet): promociones 2010 y 2011 (el cuestionario se distribuyó entre algo más de 300 residentes, de todas las especialidades). Los MIR1 de la promoción 2010 tenían la peculiaridad de proceder mayoritariamente de UNIZAR, habiendo así cursado la asignatura de bioética en su segundo curso de facultad.

No parece que haya asociación entre el C-score y la edad, aunque no tenemos demasiados casos con edad > 30 años. Tampoco hay diferencias significativas en función del sexo.

El C-score de los MIR1 es menor que el de UNIZAR: el C-score de UNIZAR antes de la asignatura de bioética $(n=273)$ muestra una media de 14,2 , mientras que el $C$-score de después $(n=194)$ tiene una media de 14,3 y los MIR1 ( $n=141)$ tienen una media de 10,4 (Kruskal-Wallis; $\chi^{2}=20,45 ; p=<0,0001$ ). No se aprecian diferencias ( $t$ test $=1,65 ; p=0,1047$ ) en cuanto al $C$-score en función de la asignatura de bioética, aunque la media es de 10,5 para los 79 de bioética frente a 8,1 para los 25 que no la cursaron (Tabla I).

Al categorizar el $C$-score en $<10$ o $\geq 10$, existe una asociación entre las proporciones de $C$-score $<10 \mathrm{y} \geq 10$ y el tipo de sujeto $\left(\chi^{2}=25,4\right.$; g.l. $=3$; $p<$ $0,0001)$. En concreto, existen diferencias sólo para UNIZAR $(Z=5,65 ; p<0,0001)$. En el resto de grupos se puede decir que las proporciones con $C$-score $<10 \mathrm{y} \geq 10$ son iguales al 50\% (Tabla II). 
$\mathrm{Al}$ analizar las respuestas de los MIR1 ante los dilemas del trabajador y del médico, se aprecia que son similares a las obtenidas entre los estudiantes de Zaragoza, sin encontrarse diferencias significativas.

\section{Discusión}

Nuestro estudio tiene una serie de limitaciones, entre ellas el bajo número de respuestas obtenidas, principalmente en Qatar, motivado en buena parte por el exiguo número total de alumnos. Para obtener una muestra representativa sería necesario continuar con el estudio durante muchos más cursos académicos dentro de la misma universidad, o bien incluir otros campus universitarios de la zona.

En el caso de UNIZAR y MIR1, aunque la asistencia a clase fue buena, superior al 50\%, y la colaboración ha sido excelente, el problema derivó de la versión validada en castellano del MJT. Si revisamos el test, nos damos cuenta de que la pregunta acerca de la opinión general ante los dilemas del trabajador y del médico se confunde fácilmente con la explicación general del significado de la escala de Likert, por lo que un porcentaje importante de discentes dejó esta pregunta en blanco, invalidando una parte muy importante de los cuestionarios, con la consiguiente pérdida de potencia estadística, incluso en el ámbito de Zaragoza.

El valor medio del C-score, como medida del razonamiento moral de los estudiantes de medicina de dos ámbitos diferentes (España, cultura occidental, y Qatar, cultura islámica) en el primer ciclo formativo, alcanza valores inusitadamente bajos, comparado con otros estudios en otros ámbitos $[6,8,11]$, confirmándose, con todo, que la media entre los MIR1 es significativamente inferior a la de UNIZAR, como en otras investigaciones realizadas $[8,11]$.

El hecho de no haber encontrado diferencias significativas entre ambas regiones, ni en el test-retest antes y después de la actividad formativa, ni en función de otras variables independientes, como el sexo y la edad, confirma la estabilidad del índice, principal indicador del MJT [17]. Por tanto, es éste un instrumento fiable que cuenta, además, con otra cualidad frente a otras herramientas de medida del razonamiento moral disponibles $[18,19]$. Tener versiones validadas en múltiples lenguas (en nuestro caso, castellano e inglés) facilita las comparaciones, además de requerir un procedimiento de aplicación sencillo, breve y eficiente para grupos numerosos de personas.

Ahora bien, que el $C$-score sea muy estable no significa que no haya diferencias entre los grupos a es-
Tabla I. Puntuaciones antes-después de la formación en bioética en Zaragoza y Qatar.

\begin{tabular}{lccccc}
\hline & $\begin{array}{c}\text { Medicina } \\
\text { Zaragoza } \\
\text { (antes) }\end{array}$ & $\begin{array}{c}\text { Medicina } \\
\text { Qatar } \\
\text { (antes) }\end{array}$ & $\begin{array}{c}\text { Medicina } \\
\text { Zaragoza } \\
\text { (después) }\end{array}$ & $\begin{array}{c}\text { Medicina } \\
\text { Qatar } \\
\text { (después) }\end{array}$ & Total \\
\hline C-score $\geq 10$ & 140 & 37 & 102 & 16 & 295 \\
& $30,0 \%$ & $7,9 \%$ & $21,8 \%$ & $34 \%$ & $63,2 \%$ \\
C-score $<10$ & $70 \%$ & $51 \%$ & $65,5 \%$ & $42 \%$ & $63 \%$ \\
\hline \multirow{2}{*}{ Total } & $12,9 \%$ & $7,71 \%$ & $11,6 \%$ & $4,7 \%$ & $36,8 \%$ \\
& $30 \%$ & $49 \%$ & $34,5 \%$ & $58 \%$ & $37 \%$ \\
\hline
\end{tabular}

Las celdas muestran $n$, porcentaje calculado sobre el total y porcentaje sobre el total por columnas.

Tabla II. C-score dicotómico $(<100 \geq 10)$ de los residentes de Zaragoza por asignatura de bioética y los estudiantes de medicina en las dos regiones.

\begin{tabular}{lccccc}
\hline & $\begin{array}{c}\text { Residentes } \\
\text { con asignatura } \\
\text { de bioética }\end{array}$ & $\begin{array}{c}\text { Residentes } \\
\text { sin asignatura } \\
\text { de bioética }\end{array}$ & $\begin{array}{c}\text { Medicina } \\
\text { Zaragoza } \\
\text { (antes) }\end{array}$ & $\begin{array}{c}\text { Medicina } \\
\text { Qatar } \\
\text { (antes) }\end{array}$ & Total \\
\hline C-score $\geq 10$ & 47 & 14 & 140 & 37 & 238 \\
& $11,5 \%$ & $3,4 \%$ & $34,2 \%$ & $9,1 \%$ & $58,2 \%$ \\
C-score $<10$ & $49 \%$ & $35 \%$ & $70 \%$ & $51 \%$ & 171 \\
& $12,0 \%$ & 26 & 60 & 36 & $41,8 \%$ \\
Total & $51 \%$ & $6,4 \%$ & $14,7 \%$ & $8,8 \%$ & $49 \%$ \\
\hline
\end{tabular}

Las celdas muestran $n$, porcentaje calculado sobre el total y porcentaje sobre el total por columnas.

tudio y que éstas no puedan valorarse mediante la aplicación del MJT, confirmando las hipótesis de partida. Así, al dicotomizar los valores de C-score obtenidos en valores bajos o muy bajos y valores medios o altos, un tercio de UNIZAR frente a la mitad de WCMC-Q obtenían puntuaciones < 10 (esto es, en fases preconvencionales de maduración de su razonamiento moral). Estas diferencias no pueden atribuirse al sexo ni a la edad, sino que dependen únicamente del distinto entorno, probablemente de las diferencias culturales entre ambas regiones.

Por otra parte, el análisis de las respuestas dadas a los dos dilemas que integran el MJT -el dilema del trabajador y el dilema del médico- constata puntuaciones inferiores en Qatar frente a Zaragoza, sin diferencias significativas en esta región entre estudiantes y residentes: los estudiantes en Qatar esta- 
ban mucho menos de acuerdo que los zaragozanos con robar el video que probaba los despidos improcedentes y, sobre todo, se mostraban totalmente opuestos a la eutanasia.

El hecho de que las opiniones pasen, sobre todo en el caso del dilema del médico y en Qatar, de valores más extremos antes de la asignatura de bioética a opciones más centrales, incluso neutras, tras la formación recibida, apoya la hipótesis de que la formación en bioética influye sobre el razonamiento moral de los estudiantes de medicina, independientemente del entorno geográfico y cultural del que procedan.

Los resultados confirman que el C-score no se modifica de forma significativa tras la actividad formativa, tanto si ésta se desarrollaba en pocas semanas o meses, como ocurrió en Qatar, como si las actividades docentes se realizaban a lo largo de todo el cuatrimestre (Zaragoza). Esto puede interpretarse en el sentido de que la asignatura de bioética por sí misma, tal y como está enfocada en los actuales planes de estudios, no es suficiente para suponer un cambio en el razonamiento moral del estudiante, lo que apoyaría la necesidad de modificar la forma de impartir dicha asignatura y, probablemente, también su contenido. Culver decía: 'el currículo de ética médica no está diseñado para mejorar el carácter moral de los futuros médicos sino para dotar, a quienes ya tienen un buen carácter, de los conocimientos intelectuales y las habilidades interaccionales que permitan que ese carácter moral se exprese en el mejor comportamiento posible' [20].

Ahora bien, la invariabilidad del C-score podría entenderse también como una ralentización de la disminución progresiva del razonamiento moral que repetidas investigaciones han constatado desde el comienzo hasta la finalización del proceso formativo de los profesionales de la medicina [8-11]. En este sentido se interpretan los hallazgos comparativos entre estudiantes y residentes en Zaragoza: menor media de C-score en los MIR1, que dicotomizada en bajo y medio, constata una influencia positiva de la formación en bioética.

Continuar con esta línea de investigación durante más años académicos y en nuevos entornos puede clarificar los hallazgos obtenidos en el presente trabajo.
Bibliografía

1. Novoa A, Molina F, Luna A. Reconstrucción del pensamiento médico: fundamentos de pragmatismo clínico. Med Clin (Barc) 2004; 123: 345-51.

2. Gracia D. Fundamentación y enseñanza de la bioética. Bogotá: El Búho; 2000.

3. Kohlberg L. Stage and sequence: the cognitive-developmental approach to socialization. In Goslin DA, ed. Handbook of socialization: theory and research. Chicago: Rand McNally; 1969.

4. Hersh R, Reimer J, Paolitto D. El crecimiento moral. De Piaget a Kohlberg. Madrid: Narcea; 1984.

5. Lind, G. The importance of role-taking opportunities for self-sustaining moral development. J Educ Res 2000; 10: 9-15.

6. Lind G. Are helpers always moral? Empirical findings from a longitudinal study of medical students in Germany. In Comunian AL, Gielen U, eds. International perspectives on human development. Lengerich: Papst Science Publishers; 2000. p. 463-77.

7. Saura-Llamas J, Sesma-Arnaiz R, Fernández-Cuenca J, Borchert-Muñoz C, Saez-Yanguas A. Postgraduate education in family education: what do we know about residents? Aten Primaria 2001; 28: 405-14.

8. Helkama K, Uutela A, Pohjanheimo E, Salminen S, Koponen A, Rantanen-Vantsi L. Moral reasoning and values in medical school: a longitudinal study in Finland. Scand J Educ Res 2003; 47: 399-411.

9. Patenaude J, Niyonsenga T, Fafard D. Changes in the components of moral reasoning during students' medical education: a pilot study. Med Educ 2003; 37: 822-9.

10. Schillinger M. Learning environment and moral development: how university education fosters moral judgment in Brazil and two German-speaking countries. Aachen: Shaker Verlag; 2006.

11. Slovácková $B$, Slovácek L. Moral judgement competence and moral attitudes of medical students. Nurs Ethics 2007; 14: 320-8.

12. Fins JJ, Gentilesco BJ, Carver A, Lister P, Acres CA, Payne R, et al. Reflective practice and palliative care education: a clerkship responds to the informal and hidden curricula. Acad Med 2003; 78: 307-12.

13. Hafferty FW. Beyond curriculum reform: confronting medicine's hidden curriculum. Acad Med 1998; 73: 403-7.

14. Hébert PC, Meslin EM, Dunn EV. Measuring the ethical sensitivity of medical students: a study at the University of Toronto. J Med Ethics 1992; 18: 142-7.

15. Self DJ, Baldwin DC Jr. Does medical education inhibit the development of moral reasoning in medical students? A cross-sectional study. Acad Med 1998; 73: 91-3.

16. Lind $\mathrm{G}$. The meaning and measurement of moral judgment competence revisited. A dual-aspect model. In Fasko D, Willis W, eds. Contemporary philosophical and psychological perspectives on moral development and education. Cresskill, NJ: Hampton Press; 2008. p. 185-220.

17. Comunian AL, Gielen UP. Promotion of moral judgement maturity through stimulation of social role-taking and social reflection: an Italian intervention study. J Moral Educ 2006; 35: 51-69.

18. Rest JR, Thoma SJ, Edwards L. Designing and validating a measure of moral judgment: stage preferences and stage consistency approaches. J Educ Psychol 1997; 89: 5-28.

19. Ishida CH. How do scores of DIT and MJT differ? A critical assessment of the use of alternative moral development scales in studies of business ethics. J Bus Ethics 2006; 67: 63-74.

20. Culver CM, Closer KD, Gert B, Brody H, Fletcher J, Jonsen A, et al. Special report. Basic curricular goals in medical ethics. N Engl J Med 1985; 312: 253-6. 